\title{
Motor cortex hyperexcitability to transcranial magnetic stimulation in Alzheimer's disease
}

\author{
V Di Lazzaro, A Oliviero, F Pilato, E Saturno, M Dileone, C Marra, A Daniele, S Ghirlanda, \\ G Gainotti, P A Tonali
}

J Neurol Neurosurg Psychiatry 2004;75:555-559. doi: 10.1136/jnnp.2003.018127

See end of article for authors' affiliations

....................

Correspondence to: Dr V Di Lazzaro, Dipartimento di Neurologia, Università Cattolica, L.go A. Gemelli 8, 00168 Rome, Italy; vdilazzaro@rm.unicatt.it

Received 6 May 2003 In revised form 30 July 2003

Accepted 6 August 2003

\begin{abstract}
Objectives: Recent transcranial magnetic stimulation (TMS) studies demonstrate that motor cortex excitability is increased in Alzheimer's disease (AD) and that intracortical inhibitory phenomena are impaired. The aim of the present study was to determine whether hyperexcitability is due to the impairment of intracortical inhibitory circuits or to an independent abnormality of excitatory circuits.

Methods: We assessed the excitability of the motor cortex with TMS in 28 patients with AD using several TMS paradigms and compared the data of cortical excitability (evaluated by measuring resting motor threshold) with the amount of motor cortex disinhibition as evaluated using the test for motor cortex cholinergic inhibition (short latency afferent inhibition) and GABAergic inhibition (short latency intracortical inhibition). The data in $A D$ patients were also compared with that from 12 age matched healthy individuals.

Results: The mean resting motor threshold was significantly lower in $A D$ patients than in controls. The amount of short latency afferent inhibition was significantly smaller in AD patients than in normal controls. There was also a tendency for $A D$ patients to have less pronounced short latency intracortical inhibition than controls, but this difference was not significant. There was no correlation between resting motor threshold and measures of either short latency afferent or intracortical inhibition $(r=-0.19$ and 0.18 respectively, NS). In 14 AD patients the electrophysiological study was repeated after a single oral dose of the cholinesterase inhibitor rivastigmine. Resting motor threshold was not significantly modified by the administration of rivastigmine. In contrast, short latency afferent inhibition from the median nerve was significantly increased by the administration of rivastigmine.

Conclusions: The change in threshold did not seem to correlate with dysfunction of inhibitory intracortical cholinergic and GABAergic circuits, nor with the central cholinergic activity. We propose that the hyperexcitability of the motor cortex is caused by an abnormality of intracortical excitatory circuits.
\end{abstract}

S everal recent studies have shown an increased excitability of the motor cortex to transcranial magnetic stimulation (TMS) in patients with Alzheimer's disease (AD). ${ }^{1-6}$ Because spinal excitability, as tested with $\mathrm{H}$ reflexes and $\mathrm{F}$ waves, is normal in AD patients, ${ }^{1}$ this effect is likely to be due to an increased excitability of motor cortical circuits in $\mathrm{AD}$.

Cortical hyperexcitability to TMS can be determined by two different mechanisms: (a) an increased excitability of the intracortical excitatory circuits, and $(b)$ an impairment of intracortical inhibitory circuits that produces an imbalance in the activity of excitatory and inhibitory circuits of the cerebral cortex, resulting in a disinhibition of the motor cortex.

Because the main excitatory neurotransmitter in the brain is glutamate, the first mechanism would imply an involvement of glutamatergic transmission in AD. Indeed, several studies suggest that abnormalities of glutamatergic neurotransmission might play an important role in $\mathrm{AD}$, and the glutamatergic hypothesis of $\mathrm{AD}$ has been proposed as an auxiliary mechanism to the cholinergic hypothesis. ${ }^{7}$

However, the hypothesis of a disinhibition of the motor cortex in $\mathrm{AD}$ should also be taken into consideration because several recent studies have demonstrated an abnormality of two inhibitory mechanisms accessible to TMS in patients with $\mathrm{AD} .{ }^{2}{ }^{4} \mathrm{In} \mathrm{AD}$ patients there is a pronounced reduction of short latency afferent inhibition (SLAI) of the motor cortex obtained by coupling peripheral nerve stimulation with TMS of the motor cortex, ${ }^{4} \mathrm{a}$ form of inhibition that is thought to depend on the integrity of central cholinergic inhibitory circuits. ${ }^{8}$ Moreover, in these patients a slight and variable reduction has been shown in short latency intracortical inhibition (SLII) produced by paired cortical stimulation. ${ }^{24}$ This latter form of inhibition is believed to involve the gamma aminobutyric acid (GABA)A activity. ${ }^{10}$

Therefore, it is not clear at present if the hyperexcitability of the motor cortex is the expression of an involvement of excitatory circuits in $\mathrm{AD}$ or if it can be explained by an impairment of inhibitory cholinergic and, to a lesser extent, GABAergic activity.

The aim of the present study was to better define the origin of the hyperexcitability of the motor cortex to TMS in AD. For this purpose, in a group of $28 \mathrm{AD}$ patients, we compared the data of cortical excitability with the amount of motor cortex disinhibition, as evaluated using the tests for SLAI and SLII.

Moreover, to further evaluate if the hyperexcitability might be related to cholinergic dysfunction, in a subgroup of $14 \mathrm{AD}$ patients we tested if it could be reversed by the administration of the cholinesterase inhibitor rivastigmine.

Abbreviations: $A D$, Alzheimer's disease; FDI, first dorsal interosseous muscle; GABA, gamma aminobutyric acid; ISI, interstimulus interval; MEP, motor evoked potential; RMT, resting motor threshold; SLAl, short latency afferent inhibition; SLIl, short latency intracortical inhibition; TMS, transcranial magnetic stimulation 


\section{METHODS \\ Patients}

We examined 28 patients with a diagnosis of probable AD according to the NINCDS-ADRDA criteria ${ }^{11}$ and 12 neurologically healthy age matched control subjects. The main clinical and demographic characteristics of $\mathrm{AD}$ patients are reported in table 1 . The mean (SD) age of the patients was 71.3 (6.8) years, while that of controls was 73.1 (5.4) years. The inclusion criteria were $(a)$ absence of other major medical illnesses and $(b)$ symptom onset no more than 5 years previously. All the AD patients selected were able to understand and carry out the simple task required for this electrophysiological study-that is, to keep fully relaxed.

In the 30 days before participating in this study, none of the patients had been treated with drugs that might have modulated cerebral cortex excitability, nor had they been treated with cholinesterase inhibitors. The study was performed according to the Declaration of Helsinki and approved by the ethics committee of the Medical Faculty of the Catholic University in Rome. Patients and their caregivers gave their informed consent before participation.

\section{Magnetic stimulation}

Magnetic stimulation was performed using two high power Magstim 200 magnetic stimulators (Magstim Co, Whitland, Dyfed, UK) connected to a Bistim module throughout all measurements.

A figure of eight coil with external loop diameters of $9 \mathrm{~cm}$ was held over the right motor cortex at the optimum scalp position to elicit motor responses in the contralateral first dorsal interosseous (FDI) muscle. The induced current flowed in a posterior-anterior direction. Surface muscle responses were obtained via two $9 \mathrm{~mm}$ diameter $\mathrm{Ag}-\mathrm{AgCl}$ electrodes with the active electrode over the motor point of the muscle and the reference on the metacarpophalangeal joint of the index finger. Muscle responses were amplified and filtered (bandwidth $3-3000 \mathrm{~Hz}$ ) by D150 amplifiers (Digitimer, Welwyn Garden City, Hertfordshire, UK). Data were collected on a computer with a sampling rate of $10 \mathrm{kHz}$ per channel and stored for later analysis using a CED 1401 A-D converter (Cambridge Electronic Design, Cambridge, UK).

\section{Motor cortex threshold}

Resting motor threshold (RMT) was defined as the minimum stimulus intensity that produced a liminal motor evoked potential (MEP) (about $50 \mu \mathrm{V}$ in $50 \%$ of 10 trials) at rest.

Table 1 Main clinical and demographic characteristics of $A D$ patients and performances at mean scores at the standard neuropsychological tasks

\begin{tabular}{lccc}
\hline & Mean (SD) & Range & Cutoff* $^{*}$ \\
\hline Age & $71.9(6.1)$ & $57-81$ & \\
Educational level & $8.23(2.3)$ & $4-17$ & \\
Time from onset (months) & $32.03(16.8)$ & $12-68$ & \\
RAVLT & & & \\
$\quad$ Immediate recall & $21.5(8.1)$ & $3-31$ & 22.63 \\
$\quad$ Delayed recall & $1.9(2.5)$ & $0-8$ & 3.09 \\
Recognition hits & $10.1(4.7)$ & $0-15$ & $>12$ \\
$\quad$ Recognition false & $10.7(8.7)$ & $0-30$ & $<2$ \\
Immediate visual memory & $14.2(3.4)$ & $4-19$ & 13.85 \\
Raven Matrices '47 & $16.3(6.45)$ & $0-33$ & 17.36 \\
Phonological Word Fluency & $14.8(9.75)$ & $2-36$ & 15.45 \\
Constructional Praxis & $56.8(11.66)$ & $21-70$ & 60.85 \\
MSE & $19.35(3.8)$ & $9-28$ & 23.7 \\
\hline
\end{tabular}

${ }^{*}$ Cutoff scores of normal controls adjusted for age and educational level ${ }^{23}$ MSE, Mini Mental State Examination
Short latency inhibition by somatosensory input from the hand

Short latency inhibition was studied using the technique that we have recently described. ${ }^{12}$ Conditioning stimuli were single pulses $(200 \mu \mathrm{s})$ of electrical stimulation applied through bipolar electrodes to the median nerve at the wrist (cathode proximal). The intensity of the conditioning stimulus was set at just over motor threshold for evoking a visible twitch of the thenar muscles. The intensity of the test cortical magnetic shock was adjusted to evoke a muscle response in relaxed FDI with an amplitude of approximately $1 \mathrm{mV}$ peak to peak.

The conditioning stimulus to the peripheral nerve preceded the magnetic test stimulus. Interstimulus intervals (ISIs) were determined relative to the latency of the N20 component of the somatosensory evoked potential induced by stimulation of the left median nerve. The active electrode for recording the N20 potential was attached $3 \mathrm{~cm}$ posterior to C3 (10-20 system) and the reference was $3 \mathrm{~cm}$ posterior to C4. Five hundred responses were averaged to identify the latency of the N20 peak. ISIs from the latency of the N20 plus $2 \mathrm{~ms}$ to the latency of the N20 plus $8 \mathrm{~ms}$ were investigated in steps of $1 \mathrm{~ms}$. Five stimuli were delivered at each ISI. The subject was given audiovisual feedback at high gain to assist in maintaining complete relaxation. The amplitude of the conditioned MEP was expressed as a percentage of the amplitude of the test MEP. We evaluated the percentage of inhibition of the conditioned responses at the seven different ISIs and averaged these values to obtain a grand mean of SLAI.

\section{Short latency intracortical inhibition}

SLII was studied using a paired pulse magnetic stimulation paradigm. ${ }^{13}$ Two magnetic stimuli were given through the same stimulating coil, using a Bistim module over the motor cortex, and the effect of the first (conditioning) stimulus on the second (test) stimulus was investigated. The conditioning stimulus was set at an intensity of 5\% (of stimulator output) below active threshold. The second (test) shock intensity was adjusted to evoke a MEP in relaxed FDI with an amplitude of approximately $1 \mathrm{mV}$ peak to peak. The timing of the conditioning shock was altered in relation to the test shock. ISIs between $1 \mathrm{~ms}$ and $3 \mathrm{~ms}$ were investigated in steps of $1 \mathrm{~ms}$. Five stimuli were delivered at each ISI. Subjects were given audiovisual feedback at high gain to assist in maintaining complete relaxation. The amplitude of the conditioned MEPs was expressed as a percentage of the amplitude of the test MEPs. Inhibition of the conditioned responses at the three different ISIs studied was averaged to give grand mean values. As there have been reports that intracortical inhibition at ISI $=1 \mathrm{~ms}$ may have a different mechanism to that at other intervals, ${ }^{14}$ we also calculated mean inhibition over ISI $=2$ and $3 \mathrm{~ms}$ only.

\section{Effects of acetylcholinesterase inhibition}

To test if RMT was sensitive to changes in ACh activity, we examined the motor threshold and the SLAI in 14 patients before and after the administration of a single dose $(3 \mathrm{mg})$ of rivastigmine, an ACh inhibitor commonly used for treatment of $\mathrm{AD}$. In 10 of these patients we also evaluated the effects of rivastigmine on SLII.

Measurements were made before and 2.4 hours after the administration, when AChE inhibition in the CSF was maximal. ${ }^{15}$

\section{Statistical analysis}

The electrophysiological parameters of $\mathrm{AD}$ patients were compared with those of controls by means of Mann-Whitney tests. Measures before and after administration of rivastigmine were 
compared by means of Wilcoxon matched pair tests. Correlations between different electrophysiological measures in patients (RMT, afferent inhibition, and intracortical inhibition) were assessed by means of Pearson's product moment correlation. All tests reported in the following are two tailed. The level of significance was set at 0.05 .

\section{RESULTS}

The N20 latency and amplitude were within normal limits in all $\mathrm{AD}$ patients. The mean (SD) RMT to TMS was significantly lower in $\mathrm{AD}$ patients than in controls (49.9 $(10.9) \% \vee 57.9$ (11.7)\%) of maximum stimulator output; $\mathrm{p}<0.05$, Mann-Whitney test, $\mathrm{N}_{1}=28, \mathrm{~N}_{2}=12$ ) (fig l).

MEPs in control subjects were inhibited when the median nerve stimulus was given before TMS of the cerebral cortex at an interval corresponding to the N20 latency +2 ms to N20 latency $+8 \mathrm{~ms}$. The amount of inhibition over this period was significantly smaller in $\mathrm{AD}$ patients than in normal controls (mean (SD) responses reduced to 86.6 (18.2)\% and 45.3 $(16.2) \%$ of test size respectively; fig 1), with a highly significant difference between the two groups $(\mathrm{p}<0.001$, Mann-Whitney test, $\mathrm{N}_{1}=28, \mathrm{~N}_{2}=12$ ). The percentages of inhibition of the conditioned responses at the individual ISIs are shown in fig 2. Patients and controls differed at all ISIs $\left(p<0.01\right.$ in each of seven Mann-Whitney tests with $\mathrm{N}_{1}=28$, $\mathrm{N}_{2}=12$ ).

There was also a tendency for $\mathrm{AD}$ patients to have less pronounced SLIIat ISIs $1-3 \mathrm{~ms}$ than controls (responses reduced to 46.8 (34.2)\% and 28.9 (12.6)\% respectively), but this difference between the two groups was not significant $\left(\mathrm{p}=0.08\right.$, Mann-Whitney test, $\left.\mathrm{N}_{1}=28, \mathrm{~N}_{2}=12\right)$ (fig 1). We also calculated mean inhibition over ISI $=2 \mathrm{~ms}$ and $3 \mathrm{~ms}$ only. The data showed the same group differences as above, with a tendency for $\mathrm{AD}$ patients to have a less pronounced SLIIat these ISIs than controls (responses reduced to 54.2 (38.6)\% and 34.7 (15.3)\% of test size respectively), but in this
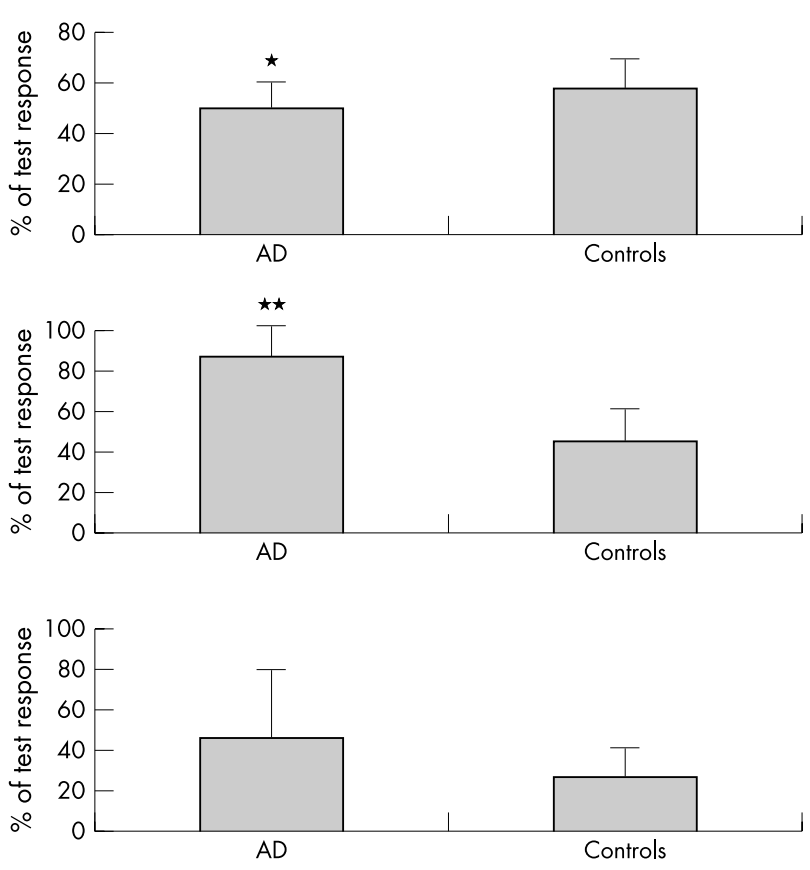

Figure 1 Resting motor threshold (top), short latency afferent inhibition (middle), and short latency intracortical inhibition (bottom) in AD patients and control subjects. Histograms show mean values, error bars are standard deviations. Resting motor threshold ( ${ }^{*} p<0.05$, MannWhitney test) and the short latency afferent inhibition $\left({ }^{* *} p<0.001\right.$, Mann-Whitney test) were significantly reduced in $A D$ patients.

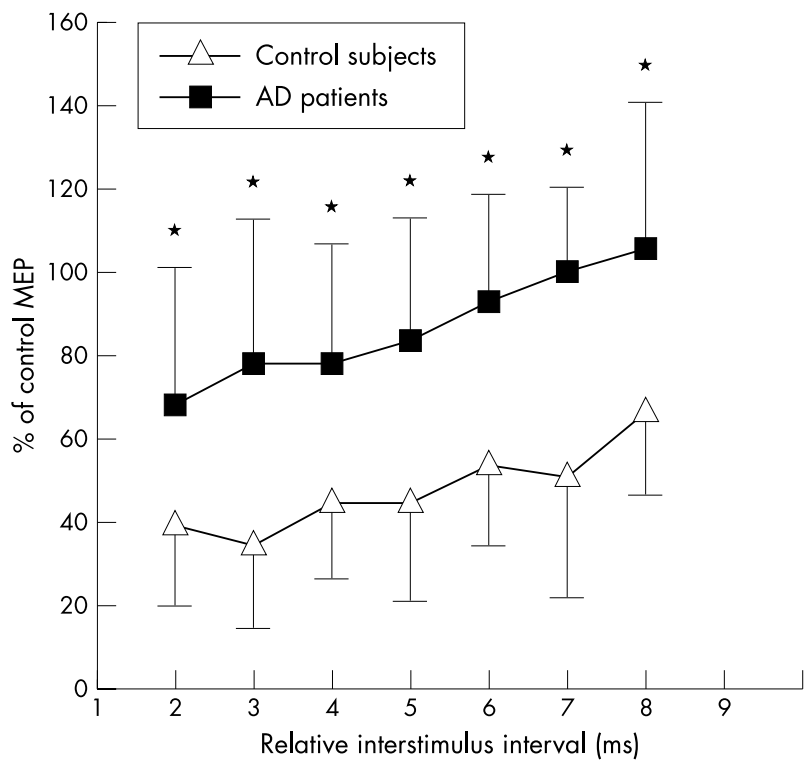

Figure 2 Short latency afferent inhibition at different interstimulus intervals in $A D$ patients (black square) and control (open triangle) subjects. The interval between median nerve stimulation and cortical stimuli has been corrected for the latency of the N20 component of the somatosensory evoked potential in each subject (see text). The size of MEPs is expressed as a percentage of the MEP evoked by magnetic stimulation alone. Short latency afferent inhibition ( ${ }^{*} p<0.01$, MannWhitney test) was significantly reduced in $A D$ patients at all explored intervals. Error bars indicate standard deviations.

case also the difference between the two groups was not significant ( $p=0.20$, Mann-Whitney test, $\mathrm{N}_{1}=28, \mathrm{~N}_{2}=12$ ).

Lastly, we found no correlation between RMT measures and measures of either short latency afferent inhibition or intracortical inhibition ( $\mathrm{r}=-0.19$ and 0.18 respectively, NS).

\section{Effects of acetylcholinesterase inhibition}

In $14 \mathrm{AD}$ patients the electrophysiological study was carried out before and after a single oral dose of the cholinesterase inhibitor rivastigmine.

RMT was not significantly modified by the administration of rivastigmine $(\mathrm{p}=0.55$, Wilcoxon matched pair test, $\mathrm{n}=14$ ) (RMT before administration, 49.9 (8.7)\%; after administration, 49.4 (9.5)\%) (fig. 2). In contrast, SLAI from the median nerve was significantly increased by the administration of rivastigmine $(\mathrm{p}=0.0 \mathrm{l}$, Wilcoxon matched pair test, $\mathrm{n}=14)$; the mean amplitudes of the conditioned response were 86.2 (16.7)\% of control size before and 70.1 $(21.2) \%$ after administration of rivastigmine (fig 3).

SLII at ISIs of $1-3 \mathrm{~ms}$ was not significantly modified by the administration of rivastigmine $(\mathrm{p}=0.64$, Wilcoxon matched pair test, $\mathrm{n}=10)$; the mean amplitudes of the conditioned response were 39.8 (26.9)\% of control size before and 35.7 (24.8)\% after administration of rivastigmine).

The mean amplitude of the muscle response evoked by the conditioning electrical stimuli to the median nerve at the wrist was not modified after rivastigmine $(\mathrm{p}=0.14$, Wilcoxon matched pair test, $\mathrm{n}=14$ ).

\section{DISCUSSION}

This study confirms that the excitability of the motor cortex to TMS is increased in AD, as demonstrated by the consistent reduction in RMT. This abnormality is associated with a pronounced abnormality of SLAI and a slight abnormality of SLII. SLAI is a test sensitive to the excitability of some cholinergic circuits in the human cerebral motor cortex ${ }^{8}$ and its abnormality is probably correlated with the most 


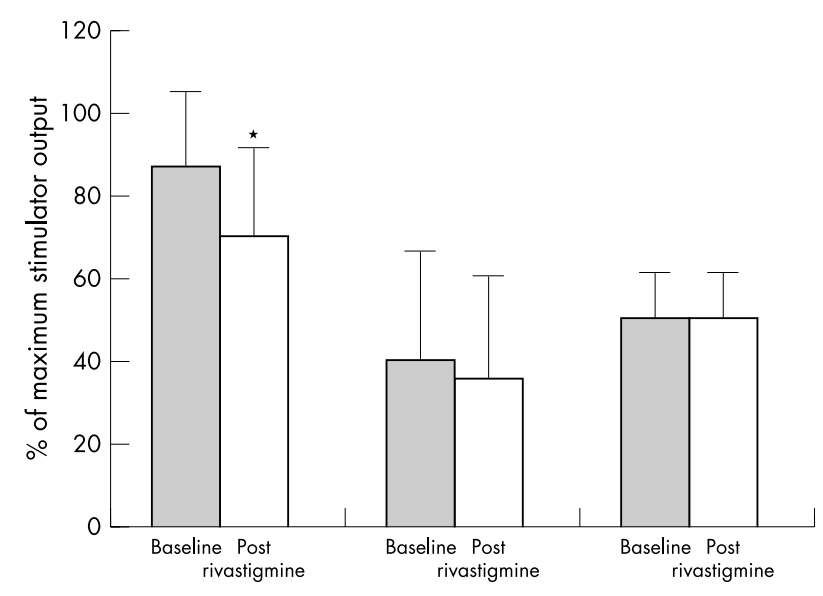

Figure 3 Effects of rivastigmine on RMT (top; 14 patients), on short latency intracortical inhibition (middle; 10 patients) and on short latency afferent inhibition (bottom; 14 patients). Histograms show mean values, error bars are standard deviations. Rivastigmine did not modify resting motor threshold and short latency intracortical inhibition but significantly increased the short latency afferent inhibition $\left({ }^{*} p=0.01\right.$, Wilcoxon matched pairs test).

consistently demonstrated deficit in AD that involves reduced cholinergic activity. However, it is still unknown if different neurotransmitters such as glutamate, GABA, or dopamine are involved in the regulation of SLAI. Therefore, we cannot exclude at present whether changes in neurotransmitter systems different from the cholinergic system can contribute to the pronounced abnormality of SLAI observed in $\mathrm{AD}$ patients.

The interpretation of the increased excitability is more complex. Is it a consequence of the impairment of inhibitory cholinergic circuits or is it the hallmark of a direct involvement of excitatory circuits? Because it has been shown that the blockade of cholinergic muscarinic receptors with scopolamine determines an increase in cortical excitability to $\mathrm{TMS}^{8}$ the first hypothesis should be taken into consideration. However, in our AD patients, RMT appears unrelated to the measure of afferent inhibition; less than $5 \%$ of the variance in RMT scores (estimated by $\mathrm{r}^{2}$, see above) could be explained by variance in afferent inhibition scores.

The effects of acetylcholinesterase inhibition on TMS parameters in a subgroup of $14 \mathrm{AD}$ patients also support the hypothesis that changes in threshold and in cholinergic circuit activity are independent. The administration of the single dose of rivastigmine did not produce any significant change in RMT. In contrast, SLAI from the median nerve was significantly increased by the administration of rivastigmine.

The reduction in RMT was not related either to the slight reduction in intracortical inhibition, a test that is sensitive to the level of GABAA activity, or to the administration of the single dose of rivastigmine, which did not produce any significant change in this parameter in $\mathrm{AD}$ patients. Therefore, the lower threshold observed in $\mathrm{AD}$ patients does not seem to be directly related to the deficit in cholinergic and GABAergic inhibition. Because it does not seem to originate from a disinhibition of the motor cortex, can the change in threshold be considered the marker of a dysfunction of the excitatory glutamatergic circuits?

A recent study by our group suggests a possible interpretation of the hyperexcitability to TMS in $\mathrm{AD} .{ }^{16}$ We have observed that a hyperexcitability of the motor cortex to TMS can be determined in normal subjects by administration of ketamine, a drug that modulates glutamatergic neurotransmission, blocks NMDA receptor activity, and enhances nonNMDA transmission through an increase in the release of endogenous glutamate. ${ }^{17}$ Because non-NMDA channels are more involved in high frequency transmission, ${ }^{18}$ the net effect of ketamine is an increase in high frequency glutamatergic neurotransmission. For this reason, ketamine determines an increase in motor cortex excitability to TMS, a technique producing a very high frequency repetitive discharge of pyramidal neurones (at around $700 \mathrm{~Hz}$ ). ${ }^{19}{ }^{20}$ The reduction in RMT in AD patients might be interpreted, in analogy with findings in normal subjects after ketamine, as the consequence of an imbalance between non-NMDA and NMDA neurotransmission in favour of the non-NMDA transmission.

This abnormality of the glutamatergic system seems to be independent from the abnormality of the cholinergic system, supporting the hypothesis that $\mathrm{AD}$ is a complex neurodegenerative disease involving several neurotransmitter systems. ${ }^{21}$

In conclusion, our study confirms that motor cortex excitability is increased in $\mathrm{AD}$ patients and suggests that this increase may reflect an imbalance in glutamatergic neurotransmission. This hypothesis raises the need for a complex strategy for the development of drugs that can modulate glutamatergic transmission, some of which are at present under study for treatment of $\mathrm{AD}{ }^{22}$ The results of the present study suggests that an ideal approach to the glutamatergic system in $\mathrm{AD}$ patients should promote an increase in NMDA transmission and a reduction in nonNMDA neurotransmission.

\section{Authors' affiliations}

V Di Lazzaro, A Oliviero, F Pilato, E Saturno, M Dileone, C Marra, A Daniele, G Gainotti, P A Tonali, Institute of Neurology, Università Cattolica, L.go A. Gemelli 8, 00168 Rome, Italy

$S$ Ghirlanda, Group for interdisciplinary cultural studies, Stockholm University, Kräftriket 7B, 10691 Stockholm, Sweden

Competing interests: none declared

\section{REFERENCES}

1 de Carvalho M, de Mendonca A, Miranda PC, et al. Magnetic stimulation in Alzheimer's disease. J Neurol 1997;244:304-7.

2 Liepert J, Bar KJ, Meske U, et al. Motor cortex disinhibition in Alzheimer's disease. Clin Neurophysiol 2001;112:1436-41.

3 Alagona G, Bella R, Ferri R, et al. Transcranial magnetic stimulation in Alzheimer disease: motor cortex excitability and cognitive severity. Neurosci Lett $2001 ; 314: 57-60$.

4 Di Lazzaro V, Oliviero A, Tonali PA, et al. Noninvasive in vivo assessment of cholinergic cortical circuits in $A D$ using transcranial magnetic stimulation. Neurology 2002;59:392-7.

5 Pennisi G, Alagona G, Ferri R, et al. Motor cortex excitability in Alzheimer disease: one year follow-up study. Neurosci Lett 2002;329:293-6.

6 Ferreri F, Pauri F, Pasqualetti P, et al. Motor cortex excitability in Alzheimer's disease: A transcranial magnetic stimulation study. Ann Neurol 2003;53:102-8.

7 Francis PT, Palmer AM, Snape M, et al. The cholinergic hypothesis of Alzheimer's disease: a review of progress. J Neurol Neurosurg Psychiatry 1999;66:137-47.

8 Di Lazzaro V Oliviero A, Profice P, et al. Muscarinic receptor blockade has differential effects on the excitability of intracortical circuits in the human motor cortex. Exp Brain Res 2000;135:455-61.

9 Ziemann U, Lonnecker S, Steinhoff BJ, et al. Effects of antiepileptic drugs on motor cortex excitability in humans: a transcranial magnetic stimulation study. Ann Neurol 1996;40:367-78.

10 Di Lazzaro V, Oliviero A, Meglio M, et al. Direct demonstration of the effect of lorazepam on the excitability of the human motor cortex. Clin Neurophysiol 2000;111:794-9.

11 McKhann G, Drachman D, Folstein M, et al. Clinical diagnosis of Alzheimer's disease: report of the NINCDS-ADRDA Work Group under the auspices of Department of Health and Human Services Task Force on Alzheimer's Disease. Neurology 1984;34:939-44.

12 Tokimura H, Di Lazzaro V, Tokimura Y, et al. Short latency inhibition of human hand motor cortex by somatosensory input from the hand. J Physiol (Lond) 2000;523:503-13.

13 Kujirai T, Caramia MD, Rothwell JC, et al. Corticocortical inhibition in human motor cortex. J Physiol Lond 1993;471:501-19.

14 Fisher RJ, Nakamura Y, Bestmann S, et al. Two phases of intracortical inhibition revealed by transcranial magnetic threshold tracking. Exp Brain Res 2002; 14:240-8.

15 Kennedy JS, Polinsky RJ, Johnson B, et al. Preferential cerebrospinal fluid acetylcholinesterase inhibition by rivastigmine in humans. J Clin Psychopharmacol 1999;19:513-21. 
16 Di Lazzaro V, Oliviero A, Profice $\mathrm{P}$, et al. Ketamine increases motor cortex excitability to transcranial magnetic stimulation. J Physiol 2003;547:485-96.

17 Moghaddam B, Adams B, Verma A, et al. Activation of glutamatergic neurotransmission by ketamine: a novel step in the pathway from NMDA receptor blockade to dopaminergic and cognitive disruptions associated with the prefrontal cortex. J Neurosci 1997; 17:2921-7.

18 Conti F, Weinberg RJ. Shaping excitation at glutamatergic synapses. Trends Neurosci 1999;22:451-8.

19 Di Lazzaro V, Oliviero A, Profice P, et al. Comparison of descending volleys evoked by transcranial magnetic and electric stimulation in conscious humans. Electroencephalogr Clin Neurophysiol 1998;109:397-401
20 Di Lazzaro V, Restuccia D, Oliviero A, et al. Effects of voluntary contraction on descending volleys evoked by transcranial stimulation in conscious humans. J Physiol 1998;508:625-33.

21 Bartus RT. On neurodegenerative diseases, models, and treatment strategies: lessons learned and lessons forgotten a generation following the cholinergic hypothesis. Exp Neurol 2000;163:495-529.

22 Kemp JA, McKernan RM. NMDA receptor pathways as drug targets. Nat Neurosci 2002;5(Suppl): 1039-42.

23 Carlesimo GA, Caltagirone C, Gainotti G. The Mental Deterioration Battery: normative data, diagnostic reliability and qualitative analyses of cognitive impairment. The Group for the Standardization of the Mental Deterioration Battery. Eur Neurol 1996;36:378-84.

\section{NEUROLOGICAL PICTURE}

\section{Scalp metastasis from glioblastoma}

A 60 year old male who had previously undergone a craniotomy and debulking of a glioblastoma multiforme re-presented with several subcutaneous nodules in his scalp. He had undergone a course of radiotherapy, and it had been one year since his original surgery. Over the two months prior to his admission he had undergone a significant decline in his level of functioning, and was now dependent for most of his activities of daily living.

Computed tomography (CT) scanning showed that the scalp lesions were vividly enhancing, and that there was diffuse intracranial recurrence, with hemispheric oedema. Owing to his poor performance status, he was treated with high dose corticosteroids, and a palliative approach was undertaken, without further surgery. The patient died two months later.

Extracranial metastasis from primary cerebral tumours is a rare occurrence. It has been reported previously with metastases to lymph nodes and via ventriculoperitoneal shunts to the abdomen, ${ }^{12}$ and also along stereotactic biopsy tracts. ${ }^{3}{ }^{4}$ Metastasis to the cranial

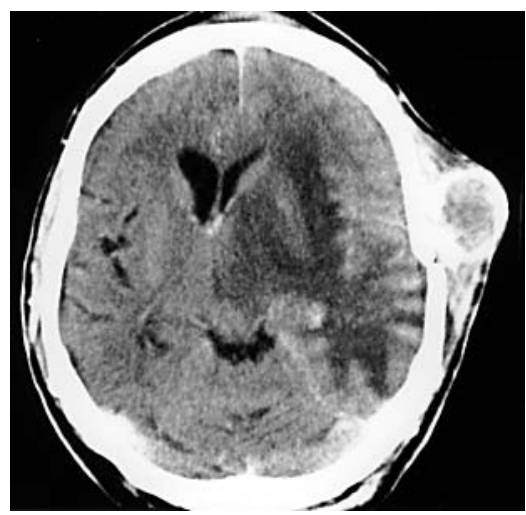

Figure 1 Post contast CT scan showing ring enhancing subcutaneous metastasis and hemispheric oedema.

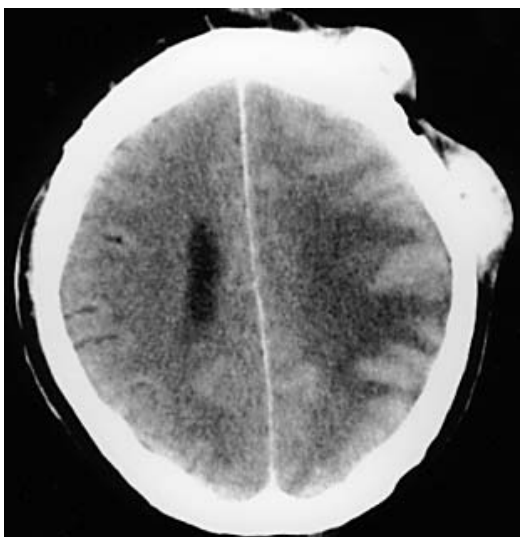

Figure 2 Post contrast CT showing two further nodules of subcutaneous tumour.

bones prior to any surgical intervention has also been documented. ${ }^{5}$ Implantation of tumour cells at the time of surgery is well described in the literature for a variety of forms of surgery, ${ }^{6}$ and there have also been occasional case reports of such metastases after neurosurgery. ${ }^{78}$ The current case serves to highlight the potential risk of such implantation and metastasis. Strategies to reduce the incidence of these metastases would include watertight approximation of the dura, replacement of the

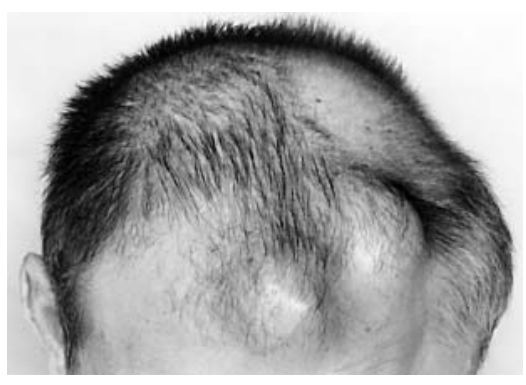

Figure 3 Posterior clinical photograph showing tumour deposits.

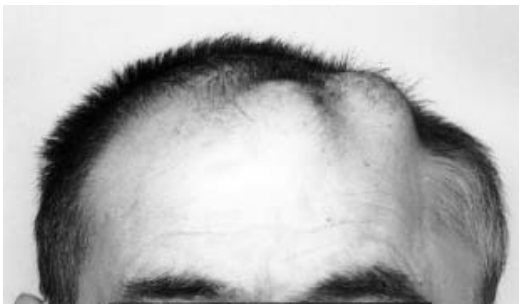

Figure 4 Anterior clinical photograph.

bone flap, and changing of surgical instruments once the intradural component of the surgery is completed.

R S Allan Westmead Hospital, Hawkesbury Road, Westmead NSW 2145, Australia; rodney@allan.com.au

\section{References}

1 Dawson TP. Pancytopaenia from a disseminated anaplastic oligodendroglioma. Neuropathology \& Applied Neurobiology 1997;23(6):516-20.

2 Pasquier B, Pasquier D, N'Golet A, et al. Extraneural metastases of astrocytomas and glioblastomas: clinicopathological study of two cases and review of literature. Cancer 1980;45(1): 112-25.

$3 \mathrm{Kim}$ JE, Kim CY, Kim DG, et al. Implantation metastasis along the stereotactic biopsy tract in anaplastic astrocytoma: a case report. Journal of Neuro-Oncology 2003;61(3):215-8.

4 Rosenfeld JV, Murphy MA, Chow CW. Implantation metastasis of pineoblastoma after stereotactic biopsy. Case report. J Neurosurg 1990;73(2):287-90

5 Choi BH, Holt JT, McDonald JV. Occult malignant astrocytoma of pons with extracranial metastasis to bone prior to craniotomy. Acta Neuropathologica 1981;54(4):269-73.

6 Bisgaard C, Jensen AS. latrogenic cutaneous implantation metastasis from exfoliated rectal adenocarcinoma cells. Case report. Acto Chirurgica Scandinavica 1989;155(2):137-8.

7 Dewar JM, Dady PJ, Balakrishnan V. Metastatic astrocytoma. Australian \& New Zealand Journal of Medicine 1985;15(6):745-7.

8 Figueroa $\mathrm{P}$, Lupton JR, Remington $\mathrm{T}$, et al. Cutaneous metastasis from an intracranial glioblastoma multiforme. Journal of the American Academy of Dermatology 2002;46(2): 297-300. 